PART II

PULSATION AND THE YOUNG DISC POPULATION 


\title{
RECENT PROGRESS IN LINEAR AND NON-LINEAR CALCULATIONS OF RADIAL STELLAR PULSATION*
}

\author{
ARTHUR N. COX \\ University of California, Los Alamos Scientific Laboratory, Los Alamos, N.M., U.S.A.
}

\begin{abstract}
This review consists of a discussion about the agreement of non-linear calculations at very low amplitude with linear theory results, the recent computational advances in progress, the current problems especially those relating to observations, a review of current computations, and finally the presentation of some recent extensive non-linear calculations.
\end{abstract}

Previous main sources of non-linear results have been Christy (1966) for RR Lyrae stars and Stobie (1969) for classical Cepheids. Now a publication by King et al. (1973) treats both linear and non-linear calculations and gives many new non-linear results.

Agreement between the results of linear calculations and those from a non-linear code when the pulsations are at very low amplitude is generally good, but there are some differences which hopefully are due to the necessary coarse zoning in the nonlinear calculations.

The best and easiest to expect agreement between the results of linear and nonlinear theories is in the pulsation periods. Extensive non-linear results have shown that non-linear period lengthening is at most $3 \%$. Thus theoretical $Q$ values from the period-mean density relation can be predicted highly accurately from linear theory. Therefore stellar masses derived from the relation between luminosity, effective temperature, period and mass, with the first three of these observed, will not be changed by non-linear theory results. Non-linear calculations are however useful in being sure that predicted linear pulsations can grow to observable amplitudes and that comparison with real observed stars is valid.

Blue edges (the high temperature boundary) of the pulsation instability strip for the fundamental and first harmonic modes have been shown to be closely the same in both the linear and the full amplitude non-linear theories, at least for Cepheids. The situation is confused somewhat for RR Lyrae stars because the two theories have not used identical opacity laws. Coarse zoning in the hydrogen ionization zone for non-linear calculations, results in either a slight under or overestimate of the instability driving due to the ionization of hydrogen. This very thin region, compared to zone sizes, generally causes an underestimate. However this small error can cause a stable model to be classed as pulsationally unstable even though it has a surface (or effective) temperature hotter than the hot (blue) edge from linear theory. Despite this calculational problem, no clear cut case has yet been found where there is a 'hard' self excited pulsation, i.e. where non-linear theory at a threshold amplitude gives pulsations when linear theory predicts stability. Since linear calculations are much easier and faster to make, it is desirable to know the limits of their usefulness in comparing with real stars.

* This work was performed under the auspices of the U.S. Atomic Energy Commission.

Ledoux et al. leds., , Stellar Instability and Erolution. 39 48. All Rights Reserved

Copyright C. 1974 by the IAU 
The phase lag of luminosity behind the time of minimum radius was originally thought to be a non-linear effect, but Castor (1968) showed that application of the proper boundary condition at the stellar surface gave the proper phase lag even in the linear theory. Non-linear effects modify the luminosity phase lag, but do not cause it.

The growth rate of the pulsation energy for all modes is the same in the linear infinitesimal amplitude theory as in the non-linear theory at very low amplitude to within $10 \%$ in the best checked case. Generally the agreement is less precise, about $50 \%$. Pulsation driving regions are balanced against the interior damping regions and the small net driving causes amplitude growth. A slight error in the amount of driving by the hydrogen ionization zone in the coarsely zoned non-linear calculations can affect the blue edge, as discussed above, as well as influence greatly the instability growth rate.

The most stringent comparison between the theories is in the behavior of $\delta r / r$, $\delta \varrho / \varrho, \delta T / T, \delta P / P$ and $\delta L / L$ throughout the unstable star model. An example of this comparison for a 6.25-day period Cepheid is shown in Figure 1 where $\delta r / r$ vs $x=r / R$ is shown at the maximum compression stage $\left(y_{1}\right)$ and then just one-quarter of a cycle

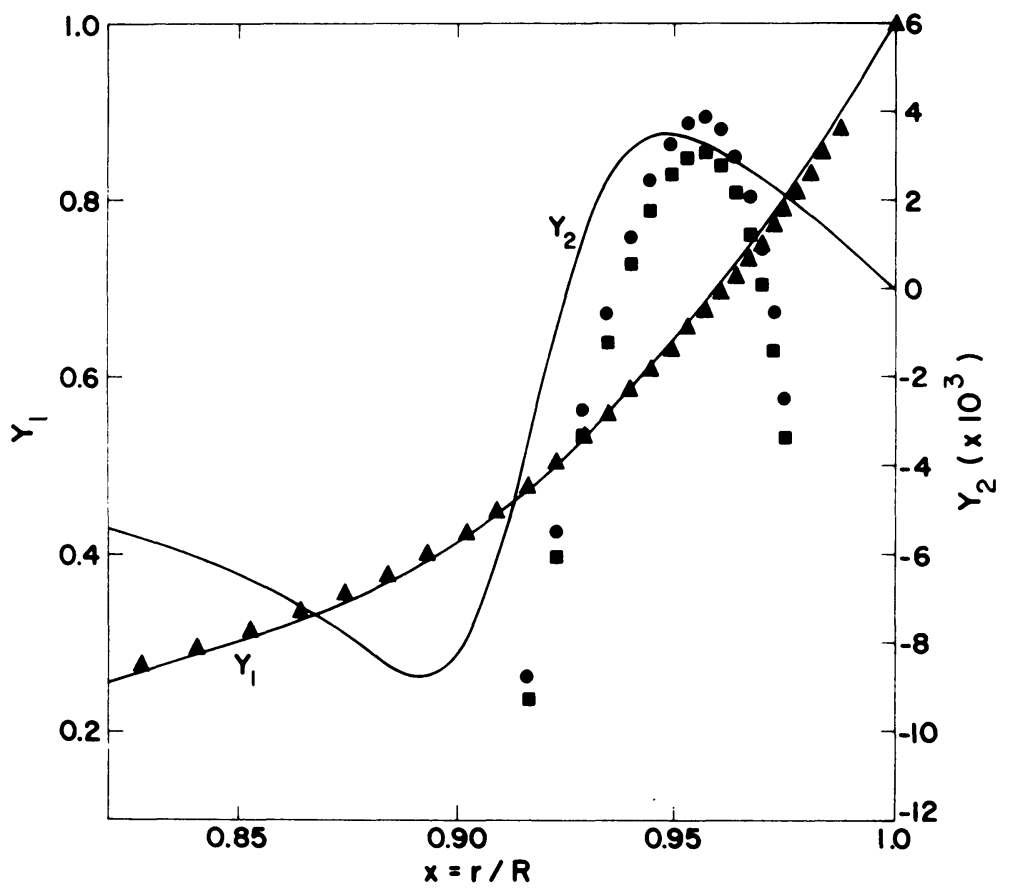

Fig. 1. The real and imaginary parts of the radius variation for a 6.25 day period 5.4 solar mass Cepheid model vs fractional radius. The $y_{1}$ linear theory result (solid line) is matched closely by the small amplitude non-linear theory results (triangles) at maximum compression. The $y_{2}$ circles and squares are at times separated by $1 / 5300$ of a period when the outer radius is at equilibrium position moving outward, and they do not agree too well with the linear theory (solid line). 
later $\left(y_{2}\right)$ when the outer radius is at its equilibrium position. The $5.4 M_{\odot}$ model used is identical in the linear and non-linear calculations, and has an equation of state allowing only the second ionization of helium to occur. The non-linear radius pulsation amplitude is 0.00123 and the variable $y$ is $\delta r / 0.00123 r$. For $y_{2}$ the dots and squares give conditions in the non-linear calculation of two times separated by $1 / 5300$ of the period centered at equilibrium radius time. While the agreement is not perfect (especially for $y_{2}$ ) the general behavior is similar enough to constitute a reasonable check of the non-linear programs at low amplitude.

There are several current, and possibly perennial, computational difficulties in both linear and non-linear work. Opacity variations with temperature and density need to be very accurately known in evaluating the stability of a stellar model. Until 1971, the usual opacity tables as published by Cox and Stewart (1970a, b), with their coarse temperature and density grid, were used. Christy (1966) fitted earlier data with a lengthy expression and was able to assure smooth variations with $T$ and $\varrho$. However, the fit is not perfect, or perhaps it no longer fits the latest opacity data. In any case the precise location of blue edges in the HR diagram depends on whether one uses the Christy formula or more recent spline fit data.

The situation seems to be that a blue edge can be reasonably located to perhaps 0.01 or better in $\log T_{\mathrm{e}}$, but the definition of the intersection luminosity of two blue edges, for example the fundamental and first harmonic blue edges, depends greatly on smooth opacity values. An accurate value of the intersection luminosity is useful in predicting possible pulsation modes.

A current calculational improvement for the radiation treatment in the outer stellar layers has been outlined by Castor (1972). Using the mean intensity as an additional variable, one can account for corrections to the radiation flow which are of the order of the material velocities divided by the speed of light. Only recent non-linear calculations by Spangenberg at Los Alamos, to be outlined later, use these latest transfer theory improvements.

Even more elaborate transport theory has been used by Keller and Mutschlecner (1970, 1971), by Bendt and Davis (1971) and by Davis $(1972,1974)$ for W Virginis. For Cepheids, it appears that one can predict spectral features such as colors at all phases of the pulsation period by merely constructing static atmospheres with effective temperature and effective gravity information from the radiation-diffusion models of Christy (1966) or most recently of King et al. (1973). Keller and Mutschlecner have shown for one Cepheid model that static atmospheres give color information identical with multi-frequency hydrodynamic transport atmospheres also. Detailed transport theory seems only to be needed for population II Cepheids such as W Virginis where the atmosphere is very extended and has shock waves passing through.

Another improvement being developed by Castor at Los Alamos is a mixed Lagrangian and Eulerian computation grid to define more accurately the very thin hydrogen ionization region. In linear calculations, it is possible to use enough thin zones to accurately define the hydrogen ionization region and its effects on pulsa- 
tional instability. In the time-consuming non-linear work, however, zones must be thicker. In the developments underway, a few thin zones can be allowed to slide through the stellar mass to follow the hydrogen ionization detail and accurately define the driving and the light curve.

Non-linear limiting amplitudes depend on the artificial viscosity used to smooth the hydrodynamic computations. This has been studied in detail in the work of Bendt and Davis (1971). When comparison of predicted amplitudes with observations are made, especially for the problem of Cepheid pulsation modes, care must be taken to avoid the strong dependence on the artificial viscosity. Generally a rather large viscosity is used to speed the calculations, and the resulting limiting amplitude behavior is not always accurately determined. Stellingwerf at Boulder has now suggested that no artificial viscosity be used except when zone compression rates exceed a specified threshold.

The theory of energy flow due to convection is always in a somewhat uncertain state in stellar interior calculations, and if the stellar structure is continually changing this time-dependent convective flux is essentially unknown. Simple phenomenological procedures have been outlined by Cox et al. (1966) and by Cox (1967), and even used for red variables by Keeley (1970) and Wood (1973). Such methods have not been used to study stars in the usual pulsation instability strip. Indeed a discussion by Castor (1971) indicates that agreement between phase shift predictions and observations seems to require lower convective flux than the current time-independent mixing length theory gives. Thus convection is usually neglected, but discussion of red edges will eventually require a theory of time-dependent convection.

The most extensive efforts at present for the improvement of non-linear calculations are development of techniques for finding non-linear periodic solutions. Baker and von Sengbusch (1969) have treated periodic pulsations as a non-linear eigenvalue problem rather than as an initial value problem. They have used a Newton-Raphson technique to adjust the radius, velocity, internal energy, and specific volume for each Lagrangian mass point at a starting phase so that after one complete period conditions repeat exactly. The procedure involves integrations through time just as the initial value problem method, but by iterations, the time integrations need only be made for as many periods as necessary until convergence to a strictly periodic solution is found. This method has been modified by Stellingwerf (1973) to make it more adaptable to the usual initial value techniques. Further following Baker and von Sengbusch, it is possible to make a linear stability analysis of the derived non-linear periodic solution to see if it will want to switch to another pulsation mode.

There is a great advantage to this technique of finding the non-linear strictly periodic solutions, because if $e$-folding times are long, direct integrations for possibly thousands of periods can be avoided. Another advantage is in the analysis to see if the solution is stable or whether it tends to a neighboring solution. However, even if the periodic solution is not stable, the change to another harmonic may not actually occur. The assumed linear perturbations to the periodic non-linear solutions may not grow to observable amplitude, and the change may not completely or even partially take place. 
A current problem relating to observations is to describe which modes actually are predicted at observable amplitudes. For example, observations indicate that first harmonic Cepheids and those with both fundamental and harmonic modes seem to be rare and at a rather short period (Fitch, 1970). It is known from linear theory for a given mass, that there is a maximum luminosity for which one can get harmonic pulsation. For higher luminosity only fundamental pulsations can occur. An accurate linear theory mapping of the HR diagram for harmonic pulsation can then help sort observations for the helium content in the ionization region if the mass (from the luminosity, effective temperature, period, mass relation) is assumed known.

Pulsation in the first harmonic mode occurs at lower luminosity than that at the intersection of the first harmonic and fundamental blue edges and to the red of the first harmonic blue edge. Even somewhat redward of the fundamental blue edge, first harmonic pulsations can persist until the fundamental growth rates from the linear theory increase with cooler temperatures to about $\frac{1}{8}$ that of the first harmonic pulsations. At this and cooler temperatures there is the possibility of either harmonic or fundamental pulsations (Christy type 3 behavior). At very cool temperatures, where King et al. (1973) get a fundamental growth rate of $\frac{1}{2}$ that of the first harmonic, only fundamental pulsation seems to exist. Mapping of these two trapsition lines which originally were thought to be almost coincident by Christy (as observations of globular cluster RR Lyrae stars seem also to show) is an important goal for non-linear studies.

For periods of Cepheids between 7 and 10 days, light and velocity curves show bumps which Christy (1968) has shown to be echoes off the hard stellar core of the previous period ionization region pressure waves. Further study by Stobie (1969) has shown that these bumps indicate low Cepheid masses, perhaps only half that given by evolutionary theory for a given luminosity. Even though these echoes seem to be correctly analyzed in terms of the mass, if luminosities and internal opacities are correct, unpublished attempts at Los Alamos to confirm this sensitivity of bump phase with mass have been unsuccessful.

A small observation-theory problem still exists in deciding how to make the proper mean of the observed color variations to plot a single position of a pulsating star on the HR diagram. A common procedure is to take the mean $B$ intensity over time and subtract the mean $V$ intensity over time and then use the $\langle B\rangle-\langle V\rangle$ in the $\mathrm{Kraft}$ (1961) relation to get $\log T_{\mathrm{e}}$. Other methods are to get $\langle B-V\rangle_{\text {mag }}$ and $\langle B-V\rangle_{\text {int }}$ where these are time means of magnitudes and intensities. It is assumed that this $\log T_{\mathrm{e}}$ is the same as in a static model before pulsations have grown to observable, non-linear amplitude. A comprehensive check of this assumption has not been published using non-linear calculation results as though they were observations.

Non-linear calculations now in progress seem to include only some conventional initial value integrations by Spangenberg at Los Alamos and at the University of Colorado and the search for periodic non-linear solutions by von Sengbusch at Munich and by Stellingwerf at the University of Colorado. Few results are currently available from the periodic solution searches, though checks of the method using Christy's RR Lyrae star models are well in hand. 
Figure 2 gives on the theoretical HR diagram small circles for luminosities and effective temperatures of $\mathbf{4 0}$ models studied for pulsation by Spangenberg using nonlinear techniques. Fundamental (FBE) and first harmonic (1HBE) blue edges are shown according to linear theory using the same material properties. Also shown is an estimated red edge (ERE) which is not possible to compute because of the earlier described convection problems. A line labelled PIM is the locus of points with the

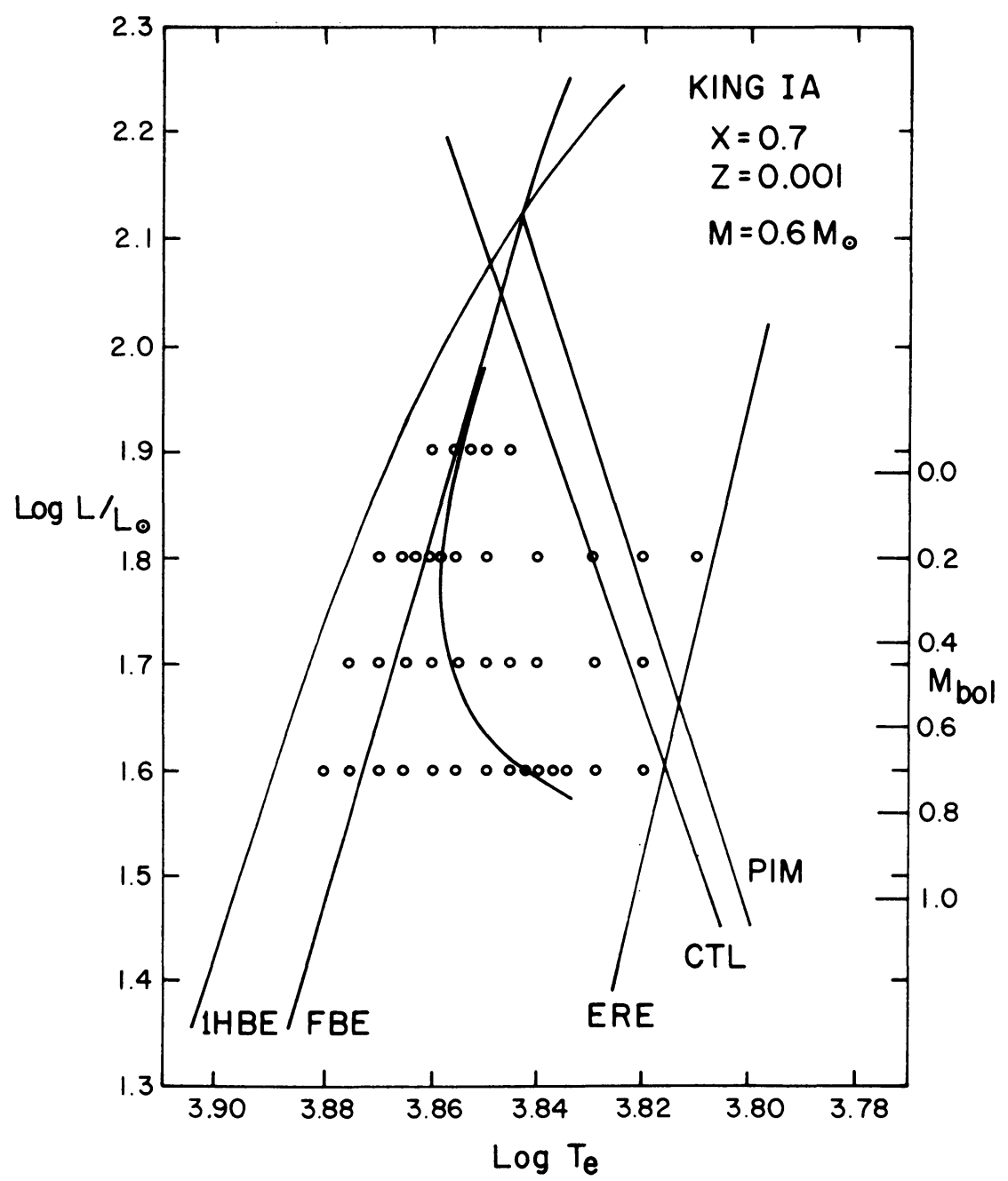

Fig. 2. The theoretical HR diagram for 0.6 solar mass models with the King Ia composition. Shown are the linear theory first harmonic blue edge (1HBE), the linear theory fundamental blue edge (FBE), the estimated red edge (ERE), The Christy transition line (CTL), the period independent of mass line (PIM), and the Spangenberg estimate of the transition line between the region of first harmonic pulsators only to pulsators with mixed harmonic and fundamental modes. The 40 models studied by the non-linear theory are given by circles for each $L, T_{\mathrm{e}}$ values. 
period independent of mass and intersecting the intersection of the first harmonic and fundamental blue edges. As plotted, this line is the $L, \log T_{\mathrm{e}}, M=0.6 M_{\odot}$ relation derived from

$$
\begin{aligned}
& L=4 \pi R^{2} \sigma T_{\mathrm{e}}^{4} \\
& Q=\pi \sqrt{ } \varrho / \varrho_{0}
\end{aligned}
$$

and the pulsation mode transition relation

$$
\log L=A \log \pi_{\mathrm{tr}}+B \text {. }
$$

Iben and Huchra (1971) have considered this PIM line as maybe the same as the Christy transition line from non-linear calculations shown as CTL with $A=1.67$ and $B=2.07$ when solar units and days are used.

For the mixture King Ia and a mass of $0.6 M_{\odot}$, these models show a curved transition line between the first harmonic oscillations and a region where both fundamental and first harmonic pulsations can exist depending upon which mode is initiated. Since integrations are carried out for only $100-1000$ periods, it is entirely possible that given the very long time for deciding a pulsation mode, a real star may eventually pulsate in a different mode than indicated on this diagram. In no case however is only fundamental pulsation seen. Thus for this mass and composition the other transition line to only fundamental pulsation does not seem to exist.

Predictions are that there is a large region where Christy type 3 behavior (both fundamental and first harmonic possible) occurs. Unfortunately in most globular clusters the transition between modes is very sharp at a given color. Only in $\omega$ Centauri does this overlap exist. Thus further theoretical work in defining the transition line is necessary.

Most recent unpublished work by Stellingwerf has shown that the artificial viscosity used in the non-linear work seems to damp preferentially the fundamental mode pulsations near the transition line. Thus the exact location and curvature of this transition line is still uncertain, but it seems to be bluer at the lower luminosities than given by Spangenberg.

In spite of extensive non-linear calculations made to date, the most pressing need is for more such studies especially to predict full amplitude behavior such as transition line positions, the significance of bumps on the light and velocity curves, harmonic and double period Cepheids. With understanding of non-linear behavior, linear small amplitude predictions can be used with more precision to study pulsating star masses and compositions.

\section{References}

Baker, N. H. and Sengbusch, K. von: 1969, Mitt. Astron. Ges. 27, 162.

Bendt, J. E. and Davis, C. G.: 1971, Astrophys. J. 169, 333.

Castor, J. I.: 1968, Astrophys. J. 154, 793.

Castor, J. I. : 1971, Astrophys. J. 166, 109.

Castor, J. I.: 1972, Astrophys. J. 178, 779.

Christy, R. F.: 1966, Astrophys. J. 144, 108. 
Christy, R. F.: 1968, Quart. J. Roy. Astron. Soc. 9, 13.

Cox, A. N. and Stewart, J. N. : 1970a, Astrophys. J. Suppl. 19, 243.

Cox, A. N. and Stewart, J. N.: 1970b, Astrophys. J. Suppl. 19, 261.

Cox, A. N., Brownlee, R. R., and Eilers, D. D. : 1966, Astrophys. J. 144, 1024.

Cox, J. P. : 1967, in R. N. Thomas (ed.), 'Cosmical Gas Dynamics' (Fifth Symposium), IAU Symp. $28,3$.

Davis, C. G.: 1972, Astrophys. J. 172, 419.

Davis, C. G. : 1974 , Astrophys. J. (in press).

Fitch, W. S.: 1970, Astrophys. J. 161, 669.

Iben, I. and Huchra, J.: 1971, Astron. Astrophys. 14, 293.

Keeley, D. A.: 1970, Astrophys. J. 161, 657.

Keller, C. F. and Mutschlecner, J. P.: 1970, Astrophys. J. 161, 217.

Keller, C. F. and Mutschlecner. J. P.: 1971, Astrophys. J. 167, 127.

King, D. S., Cox, J. P., Eilers, D. D., and Davey, W. H. : 1973, Astrophys. J. 182, 859.

Kraft, R. P.: 1961, Astrophys. J. 134, 616.

Stellingwerf, R. F.: 1973, private communication.

Stobie, R. S. : 1969, Monthly Notices Roy. Astron. Soc. 144, 485.

Wood, P. R. : 1973 (3 preprints).

\section{DISCUSSION}

Iben: Does that transition line mean that stars to the left of it pulsate solely in the first harmonic and those to the right exhibit type 3 behaviour or pulsate only in the fundamental? I do not understand your statement.

Cox: According to the Christy designation, type 1 behaviour is to the left of a transition line where only harmonic pulsation is predicted by the non-linear theory. Type 3 behaviour is the one where the full amplitude mode is either harmonic or fundamental depending on which you deliberately excite at an amplitude just below full harmonic amplitude. The line drawn is between type 1 and type 3 behaviour. Spangenberg has not yet found any cases with only the fundamental occurring - the so called type 2 behaviour.

Iben: So there is a transition region that has an edge to it? There is not a transition line?

Cox: That is correct. We don't seem to find a sharp transition line between type 1 and type 2 behaviour.

Iben: It is extremely important because in that finite sized region to the right you can have either one mode or the other and there might be a hysteresis effect and so on.

Cox: Yes, but this is not a new result. The evolution direction may determine the observed mode. Christy observes this type 3 behaviour. As a matter of fact he also observes type 4 behaviour when both modes are present simultaneously. I suppose such type 4 behaviour may occur somewhere in the type 3 behaviour region, but none have been seen by Spangenberg in this work on RR Lyrae stars.

Iben: I do believe that at one time it was suggested that there was a transition line and not a transition region and that has been the whole bone of contention for a number of years. This is the first time that it has been cleared up.

Cox: Let us look again at the luminosity-effective temperature diagram. Here is the pulsation instability strip. This is the harmonic blue edge and here to the red at low enough luminosity (below the edge intersection) is the fundamental blue edge. Even redder yet is the transition edge from type 1 to type 3 behaviour. In principle, further red is type 2 (fundamental only) behaviour but this has not been found yet by Spangenberg even very close to the estimated red edge.

It appears that there is really a transition region with type 3 and type 4 behaviour possible. How this broad region fits with the sharp transition line from observations is not clear. van Albada and Baker (Bull. A.A.S. 3, (1971), 241) suggest the importance of evolution direction, and this is further discussed without resolution by Iben (Publ. Astron. Soc. Pacific 83 (1971), 697).

Rodgers: You said earlier about the Cepheids that you were not convinced that there were any harmonic oscillators. What about the beat Cepheids? These must be in the transition zones.

Cox: What are these?

Rodgers: I call them beat Cepheids, multiple period Cepheids.

Cox: As far as I know, we have never seen Cepheids in the pure harmonic mode. We may not recognize them or they may not exist. At any rate they should be bluer and at a lower amplitude than fundamental Cepheids. Damping mechanisms limit harmonic pulsations at lower amplitude than for fundamental pulsations - just as for the c-type RR Lyrae stars. 
The beat Cepheids (type 4 behaviour) are here in my diagram. If you decrease the Cepheid mass, the harmonic and fundamental edge intersection is at a lower luminosity and harmonic pulsators would occur only at low luminosity. The same luminosity decrease of the intersection point occurs if the helium content is decreased. Thus for the beat Cepheids, there must be a type 4 region at the observed low luminosity and short period obtained either by normal helium and low mass, or at lower helium content and normal evolutionary masses. I am not sure one can ever solve the mass problem of beat Cepheids because one can not know the helium content in the helium ionization region.

Irwin: From the point of view of an observer, what does the theorist need in regard to these bumps? Does he need more information and what kind of information? More detailed information? Is it important?

Cox: I am afraid the shoe is on the other foot. I think the problem now about bumps is that we just need to sit down and do these laborious non-linear calculations with great detail. I do not think we need to have more observations. There are a number of good velocity curves, a handful, maybe half a dozen or ten, which I think is good enough at the moment. Of course, there are hundreds of light curves that have the shoulder bump but are they really precise? I am not sure. My answer to you is that I do not believe we need any more observations. We need to work harder ourselves.

Iben: Did I understand you correctly to state that there may be no first harmonic Cepheids?

Cox: I only said that because I do not know, but I would really like to hear somebody who knows the answer.

Iben: Could I show some slides that show that there may be first harmonic pulsators? I think these are Small Magellanic Cloud stars and I would like to focus on these two fellows, indicated by black filled circles, relative to the others. I would contend that these are first harmonic pulsators. (See Figure 9 in Iben's paper.)

Cox: What edges are those? Fundamental blue edges?

Iben: Yes, those are fundamental blue edges, but we all know that the Small Magellanic Cloud stars are bluer because they are metal poor. If we used the correct colour temperature conversion, all these would be over here. But the main point is that these stars are amongst the bluest of a homogenous set.

Now let us look at them in the next slide and do the same sort of comparison that one does for galactic Cepheids and note that these black fellows seem to be quite far up above the line suggested by the bulk of the Cepheids in the mass-luminosity diagram. If one now assumes that these are first harmonic pulsators instead of fundamental, which was done, they come back down sort of within the range of the others. (See Figure 8 in Iben's paper.)

Cox: These may just have more helium or something. I was hoping that you would say: here is a light curve and the amplitude is low like it is for Bailey-type-c stars or it is very symmetrical instead of askew. Maybe somebody can present those light curves?

Rodgers: I have seen Gascoigne's light curves and they are symmetrical and low amplitude.

Buscombe: The theoreticians have spoken very confidently about the left edge of this instability strip. From an observational point of view, there is something going on on the upper left of the left edge which looks like semi-periodic shock waves.

Cox: I do not understand. You mean these are more blue than the blue edge?

Buscombe: Yes, certainly. Supergiants in which there is something that repeats itself approximately in, say, 7 to 30 days.

Cox: Is there a light curve or is there just spectroscopic evidence?

Buscombe: Mostly changes in the spectrum. We will talk about that tomorrow.

Stobie: In connection with the first overtone and fundamental pulsators, there is the work of the Gaposchkins on stars in the SMC. They isolated a group in the period-luminosity plane which had sinusoidal shaped light curves of lower amplitude. It turned out that, in the L-P plane, these stars lay systematically above the other stars which have larger amplitudes and more asymmetric type of light curves. They did not say anything about this but I think this is very likely that these stars, which appear at periods less than about five days, were probably first overtone pulsators. This would agree reasonably well with the transition period which we observe in our own Galaxy among the beat Cepheids which occur in the region of two to four days that you get with both modes present.

Cox: I am glad I raised this question because I have learned there are some harmonic oscillators in the Cepheids. Are there any in our Galaxy?

Rodgers: Yes, the beat Cepheids.

Zahn: Just a comment on your comparison between linear and non-linear calculations. You seem to worry about the discrepancy that you find in $Y 2$ (see Figure 1). I think this is a genuine difference because 
it is really implied by the theory. In a non-linear calculation you have an energy source which is the $\kappa$ mechanism and a sink which is radiative damping and you have an exact balance between the source and the sink. In the linear calculations, the source predominates and the excess energy is transformed into kinetic energy to build up the amplitude. So you have a different energy flow in the two cases and you must have a difference between the linear and non-linear theories.

Cox: At least there is a great attempt in the linear theory not to get the linear expression of the differential equations but to get the linear expression of the difference equations which are used in the nonlinear calculations. There has been a great attempt to get the physics the same.

Zahn: What I am saying is that the eigenfunctions that you expect in the linear and non-linear theories must be different. At least the imaginary part, which is your $Y 2$, has to be different, otherwise the energy cannot be conserved.

Cox: When I refer to the non-linear theory, I mean using the non-linear program at low amplitude. Thus there is neither a balance of driving and damping in either theory. Hopefully they are the same with both the linear and non-linear methods, however.

Rodgers: I think part of your question was: are there any harmonic oscillators? May I address a question to Professor Eggen? Is it not true, at least among field short period Cepheids of the order of two days, that you could not put your hand on your heart and say you know the intrinsic colours well enough to say whether it is a fundamental or first harmonic oscillator?

Eggen: Yes. There are several that could be.

Cox: If there are lots of harmonic oscillators, then that means that the transition point is at a reasonable luminosity and then probably the masses of the Cepheids are - barring the fact you can trade off helium content - probably close to the evolutionary masses. 